\title{
Levantamento populacional do cervo-do-pantanal Blastocerus dichotomus (Mammalia, Cervidae) no Parque Nacional de Ilha Grande e entorno: implicações para a conservação
}

\author{
Liliani M. Tiepolo ${ }^{1}$, Walfrido M. Tomas ${ }^{2} \&$ Paulo A. Lima-Borges ${ }^{2}$ \\ 1. Universidade Federal do Paraná, Setor Litoral, Rua Jaguariaíva, 512, Caiobá, 83260-000, Matinhos, PR, Brasil. (liliani@ufpr.br) \\ 2. Embrapa/Pantanal, Rua 21 de Setembro 1880, 79320-900 Corumbá, MS, Brasil.
}

\begin{abstract}
Population survey of the marsh deer Blastocerus dichotomus (Mammalia, Cervidae) in the Ilha Grande National Park and surroundings: conservation implications. We conducted an aerial survey of the marsh deer population Blastocerus dichotomus (Illiger, 1815) in the Ilha Grande National Park and its surroundings, on the border between the states of Paraná and Mato Grosso do Sul, Brazil, during the dry season. We used the double-counting technique, resulting in a population estimate of 1079 \pm 207 marsh deers in a sampled area of $1081 \mathrm{~km}^{2}$, corresponding to an estimated density of $0.998 \pm 0.192 \mathrm{ind} / \mathrm{km}^{2}$. The population was mostly concentrated inside the park, but also occupied altered wetlands outside its limits. The results should be used to support population management and conservation measures at the national park and its surroundings, due to the enormous anthropogenic pressure upon the marsh deer population in the region.
\end{abstract}

KEYWORDS. Aerial survey, double-count technique, marsh deer, Blastocerus dichotomus, Ilha Grande National Park.

RESUMO. Nós conduzimos um levantamento populacional aéreo do cervo-do-pantanal, Blastocerus dichotomus (Illiger, 1815), no Parque Nacional de Ilha Grande e seu entorno, entre os estados do Paraná e Mato Grosso do Sul, Brasil, na estação seca. Utilizamos a técnica da contagem dupla, e a população foi estimada em $1.079 \pm 207$ cervos em uma área amostral de $1.081 \mathrm{~km}^{2}$, corresponde a uma densidade de 0,998 $\pm 0,192 \mathrm{ind} / \mathrm{km}^{2}$. A população mostrou-se mais concentrada no interior do parque, ocupando também várzeas alteradas fora dos limites do parque. Estes resultados devem subsidiar medidas de manejo e conservação da população no parque nacional e seu entorno, devido à enorme pressão antrópica sobre a população de cervos na região.

PALAVRAS-CHAVE. Levantamento aéreo, contagem dupla, cervo-do-pantanal, Blastocerus dichotomus, Parque Nacional de Ilha Grande.

O cervo-do-pantanal Blastocerus dichotomus (Illiger, 1815) é o maior representante da família Cervidae na América do Sul. A distribuição geográfica original desta espécie incluía as várzeas e planícies de inundação dos grandes rios da América do Sul e seus afluentes, a leste dos Andes, ao sul da Floresta Amazônica e ao norte da região dos Pampas, desde o sudeste do Peru até o noroeste do Uruguai e delta do Paraná (Pinder \& Grosse, 1991; Pinder \& Seal, 1994; Tomas et al., 1997; Tiepolo \& Tomas, 2006; Piovezan et al., 2010). Embora sua área de distribuição não tenha mudado muito, a intensa perda de hábitat provocou a fragmentação de suas populações (PindER \& GROSSE, 1991). Atualmente as populações mais representativas estão presentes nas bacias dos rios Araguaia, Tocantins, Xingu, Guaporé, Paraguai, Paraná e São Francisco (Tiepolo \& Tomas, 2006). No Brasil é considerado "vulnerável" (MACHADO et al., 2008), entretanto em Minas Gerais, São Paulo, Paraná e Rio Grande do Sul é classificado como "criticamente em perigo" (Machado et al., 1998; SÃo Paulo, 1998; MARQues et al., 2002; MARgarido \& Braga, 2004). A espécie é considerada extinta no Uruguai e desapareceu de grande parte de sua área de ocorrência original na Argentina, Paraguai e Peru (Tomas et al., 1997; Chebez, 2008). Globalmente é considerado "vulnerável" (IUCN, 2009) e está listado no Anexo I da Convenção sobre o Comércio Internacional de Espécies da Fauna e Flora Ameaçadas de Extinção (CITES) indicando ser espécie afetada pelo comércio ilegal de animais silvestres.
A destruição das várzeas para fins agroindustriais ou geração de energia e a caça são consideradas fatores primários de redução das suas populações. Igualmente grave é a introdução e disseminação de doenças como brucelose e febre aftosa por ungulados exóticos domésticos, conforme relatam SCHALLER \& VASCONCELOS (1978), Thornback \& Jenkins (1982), Tomas et al. (1997) e TiePolo et al. (2004). Recentemente, a construção de grandes barragens vem se tornando um dos principais fatores para o desaparecimento de populações da espécie, uma vez que eliminam as áreas de várzea, praticamente anulando qualquer possibilidade de sobrevivência e sustentabilidade de populações em longo prazo (Tomas et al., 1997; Tiepolo et al., 2004; Tiepolo \& Tomas, 2009). Outros fatores, como a drenagem de várzeas para atividades agropastoris, a precária proteção oferecida às unidades de conservação e as atividades que alteram o regime hidrológico, como hidrovias e represas, também contribuem para acelerar o processo de extinção dessa espécie (Tomas et al., 1997; TiePolo et al., 2004).

No Brasil, o cervo foi alvo de levantamentos populacionais aéreos especialmente na planície do Pantanal (Shaller \& Vasconcelos, 1978; Mauro et al., 1995; Mauro et al., 1998; Mourão et al., 2000; Tomas et al., 2001), e no rio Paraná (Mourão \& CAMPos, 1995; Pinder, 1996; ANdriolo et al., 2005). Na Argentina, BECCACECI (1994) realizou um levantamento aéreo na Reserva Natural de Iberá, também na bacia do rio Paraná. As várzeas do rio Paraná entre o Paraná e o Mato 
Grosso do Sul abrigam populações de cervos sob grande ameaça antrópica (TiEPOlo et al., 2004). O presente estudo realizou um levantamento populacional aéreo da população de cervos do Parque Nacional de Ilha Grande e seu entorno com a finalidade de subsidiar medidas para o seu manejo, monitoramento e conservação.

\section{MATERIAL E MÉTODOS}

A estimativa da população de Blastocerus dichotomus foi realizada por meio de levantamento aéreo, entre os meses de setembro e outubro de 2001, durante a estação seca, cobrindo uma área de 108.360 ha no Parque Nacional de Ilha Grande e entorno. O Parque, com uma área de 78.875 ha, localiza-se na divisa entre os estados do Paraná e Mato Grosso do Sul. Abrange áreas dos municípios de Icaraíma, Vila Alta, São Jorge do Patrocínio, Altônia e Guaíra, no Paraná, e Mundo Novo, Eldorado, Naviraí e Itaquiraí, no Mato Grosso do Sul. Inclui ainda as ilhas Grande, Peruzzi, do Pavão e Bandeirantes e todas as demais ilhas e ilhotas situadas desde o reservatório de Itaipu e da foz do rio Piquiri até a foz dos rios Amambai e Ivaí, entre as coordenadas geográficas $23^{\circ} 15^{\prime}$ a $24^{\circ} 05^{\prime} \mathrm{S}$ e $53^{\circ} 40^{\prime}$ a $54^{\circ} 17^{\prime} \mathrm{W}$ (Fig. 1).

O levantamento cobriu toda a extensão do parque e várzeas adjacentes do rio Ivaí, no Paraná, e do rio Paraná, localizadas na extensão da foz dos rios Amambai e Iguatemi, no Mato Grosso do Sul, estes fora dos limites do Parque.

Com auxílio da base cartográfica do Ministério do Exército do Brasil, em escala 1:50.000, e de uma imagem de satélite LANDSAT 7, em escala 1:25.000, foram

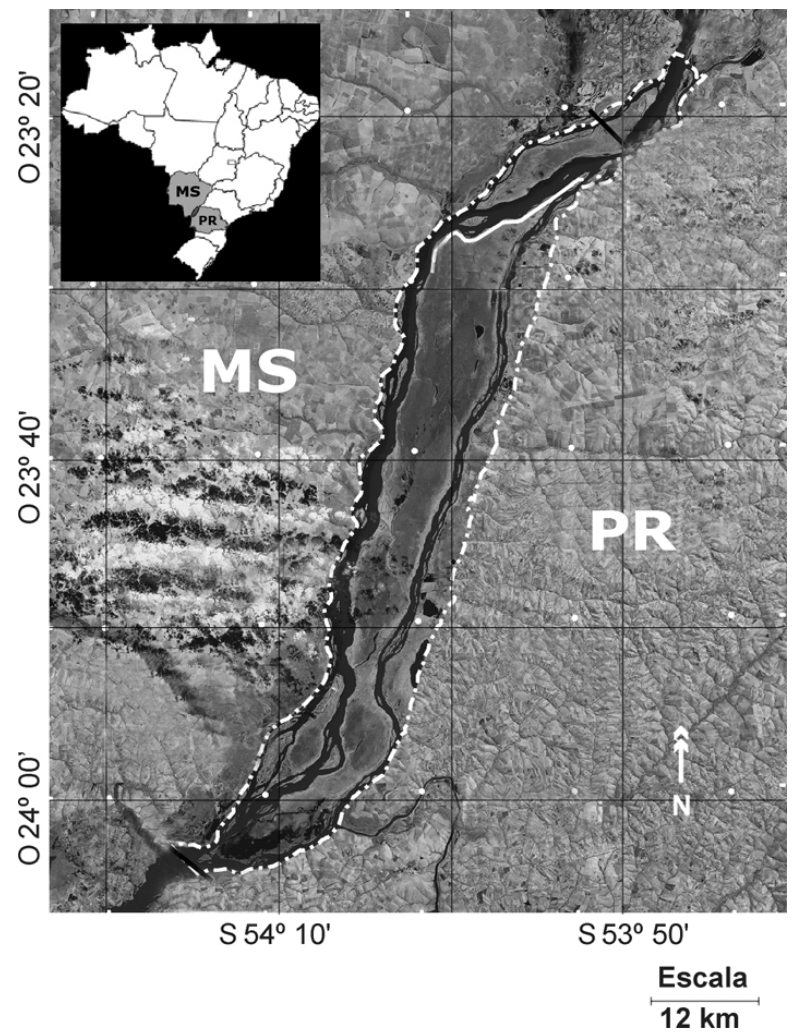

Figura 1. Localização do Parque Nacional de Ilha Grande e entorno, na divisa dos estados do Paraná e Mato Grosso do Sul, Brasil. delineadas 46 linhas de transectos cortando os gradientes das várzeas no sentido leste/oeste. A distância entre as linhas foi de $2.000 \mathrm{~m}$. Os transectos foram plotados e tiveram suas coordenadas geográficas em UTM devidamente identificadas em carta base que, juntamente com um sistema de posicionamento global (GPS), orientaram o piloto da aeronave. A área total, as áreas adjacentes sobrevoadas e a área ocupada pelo rio Paraná foram obtidas através do programa ArcView GIS 3.2.

O levantamento foi realizado com um avião CESSNA $172 \mathrm{em}$ voos de baixa altura, de aproximadamente $61 \mathrm{~m}$ em relação ao solo, e velocidade constante de $200 \mathrm{~km} / \mathrm{h}$. A configuração do voo e as marcas de visualização fixadas na asa do avião delimitaram uma faixa de contagem ao nível do solo de aproximadamente $200 \mathrm{~m}$. As 46 linhas de transectos foram subdivididas em 483 unidades de 30 segundos de voo cada uma

Para corrigir erros de visibilidade foi utilizado o método de contagem dupla (MaGnusson et al., 1978; Caughley \& Grice, 1982; Bayliss, 1986; Bayliss \& Yeomans, 1989). Assim, dois observadores contaram simultaneamente os cervos na área de amostragem, em cada unidade de 30 segundos, o que permitiu estabelecer a probabilidade de detecção de cervos por estes observadores, através da equação $\mathrm{P}=\mathrm{B} /(\mathrm{B}+\mathrm{S} 2)$ para o observador 1 , e $\mathrm{P}=\mathrm{B} /(\mathrm{B}+\mathrm{S} 1)$ para o observador 2 . Onde $\mathrm{P}=$ probabilidade de detecção; $\mathrm{B}=$ número de cervos vistos por ambos os observadores em cada unidade amostral; $\mathrm{S} 1$ = número de cervos vistos apenas pelo observador 1; $\mathrm{S} 2$ = número de cervos vistos apenas pelo observador 2 .

O cálculo das probabilidades de detecção serviu como base para a obtenção de um fator de correção a ser aplicado às contagens do observador que obteve o maior número de visualizações nos 46 transectos, onde $\mathrm{Fc}=1$ / $\mathrm{P}$, sendo $\mathrm{Fc}=$ fator de correção e $\mathrm{P}=$ probabilidade de detecção.

A estimativa populacional, densidade populacional e erro padrão foram calculados de acordo com o método de estimativa proporcional (Ratio Estimate Method) a partir do programa Survey (CAUGHLEY, 1977) onde:

$$
\mathrm{D}=\Sigma \mathrm{a} / \Sigma \mathrm{y} \quad \mathrm{e} \quad \mathrm{Y}=\mathrm{D} \text {. A }
$$

Sendo $\mathrm{a}=$ área que foi amostrada; $\mathrm{y}=$ número de cervos vistos na unidade amostral (com aplicação do fator de correção); $\mathrm{Y}=$ estimativa do número total de indivíduos na região de tamanho $\mathrm{A} ; \mathrm{D}$ = estimativa da densidade média; $\mathrm{A}$ = área total da região que foi estudada.

\section{RESULTADOS E DISCUSSÃO}

O método utilizado permitiu uma cobertura amostral de $11,26 \%$ da área total. A probabilidade de detecção de cervos obtida pelo observador 1 foi de $67 \%$, a qual resultou em um fator de correção de 1,49, que foi aplicado às contagens deste observador. A população de cervos do parque e entorno foi estimada em $1.079 \pm 207$ cervos em uma área de $1.081 \mathrm{~km}^{2}$, resultando em uma densidade de $0,998 \pm 0,192 \mathrm{ind} / \mathrm{km}^{2}$.

A razão sexual não pode ser estimada porque em muitos casos os machos adultos perdem a galhada, além de machos jovens e sub-adultos ainda não possuírem esta estrutura característica, o que pode ocasionar em 
erro de identificação, subestimando os machos ou superestimando as fêmeas da população. PINDER (1997) também percebeu este problema, encontrando um número reduzido de machos em relação às fêmeas em um levantamento aéreo por ele conduzido.

O resultado demonstra que a ilha Grande concentra o maior número de cervos, comprovando ser o local mais importante para se adotar medidas de manejo e proteção como, por exemplo, intensificar a fiscalização e implantar um programa de monitoramento. Esta é a região do parque que se apresenta em melhor estado de conservação, uma vez que as várzeas nas margens do rio Paraná no Mato Grosso do Sul e no Paraná estão deterioradas por drenagens para aproveitamento agrícola e pecuário. Também é possível detectar que a densidade de cervos difere entre os locais analisados. Por exemplo, a várzea da lagoa Xambrê, situada na margem paranaense, ao sul, no município de Altônia, suporta uma densidade de 1,5 $\mathrm{ind} / \mathrm{km}^{2}$. Embora toda a várzea faça parte do parque, nessa porção de $4,1 \mathrm{~km}^{2}$ houve uma forte intervenção da promotoria pública do município de Altônia, proibindo o uso da área pelos criadores de gado que a utilizavam como pasto de forma ilegal. $\mathrm{O}$ mesmo não ocorreu nos outros dois municípios por onde esta várzea se estende (São Jorge do Patrocínio e Vila Alta). No restante da área, com aproximadamente $9,22 \mathrm{~km}^{2}$, a densidade foi de apenas $0,1 \mathrm{ind} / \mathrm{km}^{2}$.

Outro exemplo refere-se à ilha Bandeirantes, que apresentou densidade de $0,32 \mathrm{ind} / \mathrm{km}^{2}$. Essa ilha teve um histórico de ocupação antrópica mais intenso e atualmente uma rodovia federal corta a ilha no terço superior, degradando ainda mais suas várzeas, o que pode explicar a baixa densidade de cervos observada.

A estimativa de Mourão \& CAmpos (1995) na área que foi inundada pelo reservatório da UHE de Porto Primavera, no rio Paraná, foi de 650 cervos para uma área de $1.280 \mathrm{~km}^{2}$, o que resultava uma densidade de $0,51 \mathrm{ind} /$ $\mathrm{km}^{2}$ antes do enchimento do reservatório. PINDER (1996) obteve uma média de 940 cervos para uma área de 2.500 $\mathrm{km}^{2}$, na mesma região, obtendo uma densidade de 0,37 $\mathrm{ind} / \mathrm{km}^{2}$. No entanto, ambos não estimaram as pequenas populações dos tributários do rio Paraná, como os rios do Pântano, Pardo, Verde e Aguapeí. Um estudo mais recente, conduzido por ANDRIOLO et al. (2005), estimou 896 cervos para uma área de 180.000 ha, resultando em 0,49 ind $/ \mathrm{km}^{2}$ pós-enchimento da represa de Porto Primavera.
Ainda na bacia do rio Paraná, BECCACECI (1994) estimou a população de cervos na Reserva Natural de Iberá, na Argentina, em 1.100 indivíduos em uma área de $12.000 \mathrm{~km}^{2}$, resultando em uma densidade de apenas $0,09 \mathrm{ind} / \mathrm{km}^{2}$.

Considerando que uma parte significativa da população estimada por Mourão \& CAMPOS (1995) e PINDER (1996) foi extinta em decorrência do enchimento do reservatório da UHE de Porto Primavera, ocorrido a partir de 1999, os cervos estimados para o Parque Nacional de Ilha Grande e aqueles do Parque Estadual das Várzeas do Rio Ivinhema representam a maior população remanescente de cervos na bacia do rio Paraná em território brasileiro.

Para o Pantanal, o trabalho pioneiro de Schaller \& VASCONCELOS (1978) obteve a estimativa de 7.000 cervos em uma área de $140.000 \mathrm{~km}^{2}$. Também no Pantanal, utilizando técnicas mais acuradas para o levantamento com o uso de fatores de correção para erros de visibilidade, Mourão et al. (2000) estimaram densidades de jacaré-do-pantanal (Caiman yacare Daudin, 1802), cervo e veado-campeiro (Ozotoceros bezoarticus Linnaeus, 1758) entre 1991 e 1993 em 11 sub-regiões do Pantanal, cobrindo uma área de $136.927 \mathrm{~km}^{2}$. SCHALLER \& VASCONCELOS (1978) apresentaram estimativas de densidade de cervo para diferentes áreas do Pantanal, demonstrando que a heterogeneidade de ambientes existentes na planície influencia a densidade de cervos. As densidades por eles obtidas variaram de $0,6 \mathrm{ind} / \mathrm{km}^{2}$ na área do rio Taquari a $0,26 \mathrm{ind} / \mathrm{km}^{2}$ na área do Pantanal Norte. Entretanto Mourão et al. (2000) obtiveram densidades mais altas de cervos no rio Paraguai $(0,98 /$ $\left.\mathrm{km}^{2}\right)$, na região do Corixo Grande $\left(0,78 / \mathrm{km}^{2}\right)$ e em Aquidauana / rio Negro $\left(0,57 / \mathrm{km}^{2}\right)$.

Tomas et al. (2001) realizaram contagens em períodos de seca e cheia na região do rio Negro, no Pantanal, Mato Grosso do Sul, apontando que as densidades variam não apenas em função da época do ano, mas em função da distribuição espacial dos ambientes adequados na área. As densidades neste estudo variaram de 0,53 a $1,85 \mathrm{ind} / \mathrm{km}^{2}$ nos períodos mais secos, não se detectando cervos nas áreas mais altas e naquelas de águas mais profundas, e 0,24 a $0,73 \mathrm{ind} / \mathrm{km}^{2}$ durante as cheias, não se detectando cervos nas áreas de alta inundação. As informações sobre levantamentos populacionais de cervos realizados na América do Sul são sintetizadas na tabela I.

Tabela I. Estimativas populacionais e densidade (D) de Blastocerus dichotomus (Illiger, 1815) no Brasil e Argentina.

\begin{tabular}{|c|c|c|c|c|}
\hline Local & Área $\left(\mathrm{km}^{2}\right)$ & População & $\mathrm{D}\left(\mathrm{ind} / \mathrm{km}^{2}\right)$ & Fonte \\
\hline Parque Nacional de Ilha Grande e entorno & 1.081 & $1.079 \pm 207$ & $0,998 \pm 0,192$ & presente estudo \\
\hline Pantanal Mato-Grossense & 140.000 & 7.000 & 0,26 a 0,6 & Schaller \& VASCONCELOS (1978) \\
\hline $\begin{array}{l}\text { Estero del Iberá, Reserva Natural del Ibera, } \\
\text { Corrientes, Argentina }\end{array}$ & 12.000 & 1.100 & 0,09 & ВЕССАCECI (1994) \\
\hline $\begin{array}{l}\text { Rio Paraná, montante da Usina Hidrelétrica de Porto } \\
\text { Primavera, entre São Paulo e Mato Grosso do Sul }\end{array}$ & 1.280 & 650 & 0,51 & MOURÃO \& CAMPOS (1995) \\
\hline $\begin{array}{l}\text { Rio Paraná, montante da Usina Hidrelétrica de Porto } \\
\text { Primavera, entre São Paulo e Mato Grosso do Sul }\end{array}$ & 2.500 & 940 & 0,37 & PINDER (1996) \\
\hline Pantanal Mato-Grossense & 136.927 & $44.000 \pm 7700$ & 0,10 a 0,98 & MOURÃo et al. (2000) \\
\hline Pantanal do Rio Negro, Mato Grosso do Sul & 760 & $\begin{array}{l}290 \pm 275 \\
300 \pm 109\end{array}$ & 0,53 a 1,85 & TomAs et al. (2001) \\
\hline Rio Paraná, montante da UHE Porto Primavera & 1.800 & 896 & 0,49 & ANDRIOLO et al. (2005) \\
\hline
\end{tabular}


O que estes resultados evidenciam é que a movimentação dos cervos deve ser levada em consideração para o delineamento de unidades de conservação. Trazendo esta questão para o manejo de áreas protegidas, o Parque Nacional de Ilha Grande é um exemplo de unidade de conservação que foi criada sem levar em consideração as características do hábitat e das espécies que se desejava conservar. A conservação dos últimos remanescentes das várzeas da bacia do rio Paraná foi utilizada para justificar a criação do parque nacional e nessa ocasião poderiam ter sido incorporadas aos seus limites as importantes várzeas do rio Ivaí, no estado do Paraná, e dos rios Iguatemi e Amambai, no estado do Mato Grosso do Sul. A efetiva proteção dessas várzeas, por exemplo, é indispensável para a conexão entre os cervos de Ilha Grande e os do rio Ivinhema, além de aumentar a capacidade de suporte (TIEPOLO et al., 2004).

Uma observação atenta dos limites do parque (Fig. 1) remete a um problema grave que ocorre tanto nas épocas de cheias, agora controladas artificialmente pelas inúmeras usinas de geração de energia existentes no rio Paraná, quanto nas épocas secas, quando aumenta consideravelmente a incidência de incêndios nas várzeas (Tiepolo et al., 2004). O cervo-do-pantanal, embora adaptado a viver em ambientes pantanosos e com capacidade física para escapar destes incêndios atravessando o rio Paraná, não encontra áreas seguras e protegidas nas margens, tornando-se alvo fácil de caçadores e também de cães domésticos. Esta situação é particularmente grave no sul e no norte da ilha e na margem direita (Mato Grosso do Sul) do rio Paraná. Ao sul da ilha, os cervos podem escapar para as várzeas do rio Iguatemi e ao norte para as várzeas do rio Amambai, ambas na margem direita do rio Paraná, no entanto essas áreas sofrem intensa pressão de caça (TiEPolo et al., 2004), o que pode ser a razão da baixa densidade de cervos observada.

O cenário da população de cervos do Parque Nacional de Ilha Grande é de crítico perigo de extinção, uma vez que a unidade de conservação não consegue protegê-la em seus limites (TiEPOLO et al., 2004). Agravase a situação quando os resultados deste trabalho revelam que a maior parte da população encontra-se dentro da ilha, estando sujeita aos efeitos deletérios de populações pequenas e fragmentadas. Neste caso as variações ambientais, demográficas e genéticas podem colocar esta população em grave risco de extinção em curto período de tempo. Este trabalho, portanto, evidencia que são necessárias e urgentes medidas de manejo como a ampliação dos limites do parque; o estudo do comportamento do fogo e a intensificação de proteção à espécie durante períodos de incêndios; o monitoramento contínuo da população; programas de educação e sensibilização ambiental que incluam as populações das cidades vizinhas ao parque na conservação das várzeas protegidas e suas espécies; buscar mecanismos para eliminar as modalidades de caça na região bem como prevenir atropelamentos nas estradas e rodovias locais; entre outras previstas no Plano de Conservação para a espécie (TiEPOlo \& Tomas, 2009). No entanto, estas medidas perdem efeito sem mecanismos eficientes de gestão socioambiental na unidade de conservação. O envolvimento dos diferentes atores sociais locais com a espécie e seu hábitat são imprescindíveis para o entendimento da necessidade de conservação do Parque Nacional de Ilha Grande. Conciliar os interesses sociais, econômicos, políticos e ambientais buscando eliminar conflitos; observar o cumprimento da legislação vigente; prevenir e minimizar impactos ambientais; facilitar a aplicação de recursos financeiros para conservação; ampliar as políticas públicas de conservação e proteção da biodiversidade; e promover a melhoria da qualidade de vida das populações que vivem no entorno das unidades de conservação é hoje o grande desafio da gestão ambiental para a conservação das paisagens naturais e da biodiversidade do Brasil.

Agradecimentos. Ao CNPq pela Bolsa de Mestrado concedida à primeira autora junto à Pós-Graduacão em Engenharia Florestal da Universidade Federal do Paraná. Á Fundação Dalmo Giacometti pelo financiamento dos sobrevoos. Ao apoio do piloto Celso Lombriller (Viaer - Umuarama) na condução da aeronave. A Carlos Firkowski, Miguel Milano, Fernando Fernandez, Tereza Cristina Castellano Margarido, Juliana Quadros, Paulo Henrique Carneiro Marques e Antonio Serbena pelas contribuições ao manuscrito. Ao Parque Nacional de Ilha Grande, na pessoa de Maude Nancy Joslin Motta, e a todos da equipe de gestão e apoio. A Luigi M. Melnechuky pela ajuda na confecção da imagem da área de estudo. À Sociedade Fritz Müller de Ciências Naturais e todos seus integrantes pelo apoio logístico.

\section{REFERÊNCIAS BIBLIOGRÁFICAS}

Andriolo, A.; Piovezan, U.; Costa, M. J. R. P.; LaAke, J. \& Duarte, J. M. B. 2005. Aerial line transect survey to estimate abundance of marsh deer (Blastocerus dichotomus) (Illiger, 1815). Brazilian Archives of Biology and Technology 48(5):807-814.

BAYLISS, P. 1986. Factors affecting aerial surveys of marine fauna, and their relationship to a census of dugongs in the coastal waters of Northern Territory. Austrian Wildlife Resource 13:27-37

Bayliss, P. \& Yeomans, K. M. 1989. Correcting bias in aerial survey population estimates of feral livestock in Northern Australia using double count technique. Journal of Applied Ecology 26:925-933.

BeCCACECI, M. D. 1994. A census of marsh deer in Iberá Natural Reserve, its Argentine stronhold. Oryx 28:131-134.

Caughley, G. 1977. Analysis of vertebrate populations. New York, Wiley. 234p.

Caughley, G. \& Grice, D. 1982. A correction factor for counting emus from the air, and its application to counts in Western Australia. Australian Wildlife Resource 9:252-259

Chebez, J. C. 2008. Los que se van: especies argentinas em peligro. Mamíferos. Buenos Aires, Albatroz. v.3, 336p.

IUCN. 2009. The IUCN Red List of Threatened Animals Disponível em: <http://www.redlist.org>. Acesso em: 13.01.2010.

Machado, A. B. M.; Drummond, G. M. \& Paglia, A. P. 2008. Livro vermelho da fauna brasileira ameaçada de extinção. Brasília, Ministério do Meio Ambiente / Fundação Biodiversitas. v.2, 1420p.

Machado, A. B. M.; Fonseca, G. A. B.; Machado, R. B.; Aguiar, L. M. S. \& Lins, L. V. 1998. Livro vermelho das espécies ameaçadas de extinção da fauna de Minas Gerais. Belo Horizonte, Fundação Biodivesitas. 605p.

Magnusson, W. E.; Caughley, G. J. \& Grigg, G. C. 1978. A double survey estimate of population sizes from incomplete counts. Journal of Wildlife Management 42:174-176.

Margarido, T. C. C. \& Braga, F. G. 2004. Mamíferos. In: Мikich, S. B. \& BÉrnILS, R. S. eds. Lista vermelha de animais ameaçados de extinção no Estado do Paraná. Curitiba Instituto Ambiental do Paraná / Deutsche Gesselschaft für Technische Zussammenarbeit. p.27-142. 
Marques, A. A. B.; Fontana, C. S.; Vélez, E.; Bencke, G. A.; Scheneider, M. \& Reis, R. E. 2002. Lista das espécies da fauna ameaçadas de extinção no Rio Grande do Sul. Decreto $\mathrm{n}^{\circ}$ 41.672, de 11 de julho de 2002. Porto Alegre, FZB/MCT-PUC-RS/PANGEA. 52p.

Mauro, R. A.; Coutinho, G. M.; Silva, M. E. \& Magnusson, W. E. 1998. Abundance and distribution of marsh deer Blastocerus dichotomus (Artiodactyla, Cervidae) in the Pantanal, Brazil. Revista de Ecología Latino Americana 5(1-2):13-20.

Mauro, R. A.; Mourão, G. M.; Silva, M. P.; Coutinho, M. E.; Tomas, W. M. \& Magnusson, W. W. 1995. Influência do hábitat na densidade e distribuição de cervo (Blastocerus dichotomus) durante a estação seca, no Pantanal Matogrossense. Revista Brasileira de Biologia 55(4):745-751.

Mourão, G. M. \& CAmpos, Z. 1995. Survey of broad-snouted caiman Caiman latirostris, marsh deer Blastocerus dichotomus and capybara Hydrochaeris hydrochaeris in the area to be inundated by Porto Primavera Dam, Brazil. Biological Conservation 73:27-31.

Mourão, G. M.; Coutinho, M.; Mauro, R; Campos, Z.; Tomas, W. M. \& Magnusson, W. 2000. Aerial surveys of caiman, marsh deer and pampas deer in the Pantanal Wetland of Brazil. Biological Conservation 92(2): 175-183.

Pinder, L. 1996. Marsh deer population estimate in the Paraná river, Brazil. Biological Conservation 75:87-91.

1997. Estimativa da população de cervos-do-pantanal no rio Paraná utilizando levantamento aéreo. In: VAlladAREsPadua, C. \& Bodmer, R. E. orgs. Manejo e Conservação de Vida Silvestre no Brasil. Belém, Sociedade Civel Mamirauá. p.116-122.

Pinder, L. \& Grosse, A. P. 1991. Blastocerus dichotomus. Mammalian Species 380:1-4.

Pinder, L. \& SEAL, U. S. 1994. Cervo-do-pantanal Blastocerus dichotomus análise da viabilidade de população e hábitat (PHVA). Apple Valley, IUCN/SSC Conservation Breending Specialist Group. 172p.
Piovezan, U.; Tiepolo, L. M.; Tomas, W. M.; Duarte, J. M. B.; VAREla, D. \& Marinho-Filho, J. S. 2010. Marsh Deer Blastocerus dichotomus (Illiger, 1815). In: Duarte, J. M. B. \& GonzÁlez, S. eds. Neotropical Cervidology: Biology and Medicine of Latin American Deer. Jaboticabal, Funep/IUCN. p.66-76

São PAulo (Secretaria de Estado do Meio Ambiente). 1998. Fauna Ameaçada do Estado de São Paulo. São Paulo, SMA/CED. $60 \mathrm{p}$.

Schaller, G. B. \& Vasconcelos, J. M. 1978. A marsh deer census in Brazil. Oryx 14:345-351.

Thornback, J. \& Jenkins, M. 1982. The IUCN Mammal Red Data Book. Parte II. Gland, Switzerland, International Union for Conservation of Nature. 516p.

Tiepolo, L. M.; Fernandez, F. A. S. \& Tomas, W. M. 2004. A conservação da população de cervo-do-pantanal Blastocerus dichotomus (Illiger, 1815) (Mammalia, Cevidae) no Parque Nacional de Ilha Grande e entorno. Revista Brasileira de Conservação da Natureza 2(1):56-66.

Tiepolo, L. M. \& Tomas, W. M. 2006. Ordem Artiodactyla. In: Reis, N. R.; Peracchi, A. L.; Pedro, W. A. \& Lima, I. P. eds Mamíferos do Brasil. Londrina, Universidade Estadual de Londrina. p.283-303.

2009. Plano de Conservação para o cervo-do-pantanal (Blastocerus dichotomus). In: Vidolin, G. P.; Tussolino, M. DE G. P. \& BRitTo, M. DE M. orgs. Planos de Conservação para Espécies de Mamíferos Ameaçadas. Curitiba, Instituto Ambiental do Paraná/Projeto Paraná Biodiversidade. p.176-201.

Tomas, W. M.; Beccaceci, M. D. \& Pinder, L. 1997. Cervo-dopantanal Blastocerus dichotomus. In: DuARTE, J, M. B. ed. Biologia e conservação de cervídeos sul-americanos: Blastocerus, Ozotoceros e Mazama. Jaboticabal, FUNEP. p. 24-40.

Tomas, W. M.; Salis, S. M.; Silva, M. P. \& Mourão, G. M. 2001. Distribution of Marsh deer (Blastocerus dichotomus) as a function of the floods in the Pantanal wetland, Brazil. Studies on Neotropical Fauna and Environment 36(1):9-13.

Recebido em janeiro de 2007. Aceito em janeiro de 2010. ISSN 0073-4721

Artigo disponível em: www.scielo.br/isz

Iheringia, Sér. Zool., Porto Alegre, 100(2):111-115, 30 de junho de 2010 